\title{
EMG Artifacts Removal from Multi-Channel EEG Signals using Multi-Channel Singular Spectrum Analysis
}

\author{
$1^{\text {st }}$ Muhammad Zubair \\ Department of Electronics and Communication Engineering \\ Prasad V. Potluri Siddhartha Institute of Technology \\ Vijayawada, Andhra Pradesh, India \\ muhammadzub17@gmail.com
}

\author{
$2^{\text {nd }}$ Umesh Kumar Naik M \\ Department of Electronics and Communication Engineering \\ Prasad V. Potluri Siddhartha Institute of Technology \\ Vijayawada, Andhra Pradesh, India \\ umeshkumar.mudavath@gmail.com
}

\author{
$3^{\text {rd }}$ Rafi Ahamed Shaik \\ Department of Electronics and Electrical Engineering \\ Indian Institute of Technology Guwahati \\ Guwahati, Assam, India \\ rafiahamed@iitg.ac.in
}

\begin{abstract}
The Electroencephalogram (EEG) is the brain signals which are most normally debased by Electromyogram (EMG) antiquities. The presence of these EMG antiquities covers the necessary information in an EEG signal. In this paper, we have proposed another strategy named as Multi-channel Singular Spectrum Analysis (MSSA) in light of Singular Value Decomposition (SVD) to expel muscle or EMG antiquities from multi-channel EEG signals. At first, the orthogonal eigenvectors of multi-channel data are estimated by performing SVD which are acquired from the covariance matrix . Since the frequency variations of eigenvectors related to EEG signal are quite low when compared to the EMG signal, so we fix some peak frequency threshold to find out the frequencies related to EEG signal, then the frequencies related to EMG signals are suppressed and the artifact free Multi-channel EEG signal is extracted. Finally, our proposed technique is applied on a noisy sinusoidal signals to test the performance of the proposed method and then it is applied on synthetic EEG signals mixed with the EMG artifacts. Simulation results are then compared with Canonical Correlation Analysis (CCA) to show that the proposed method eliminates EMG antiquities more adequately without amending the required data.
\end{abstract}

Index Terms-- Electroencephalogram (EEG), Electromyogram (EMG), Multichannel Singular Spectrum Analysis (MSSA), Singular Value Decomposition (SVD) and Canonical Correlation Analysis (CCA).

\section{INTRODUCTION}

An electroencephalogram (EEG) is a graphic record of the activity of a huge number of neuronal membrane potentials. It is widely used for the diagnosis, monitoring, and management of neurological disorders. "They are often contaminated by antiquities such as Electromyogram (EMG) signals, which are caused by the muscle contraction due to frowning, biting or chewing" [1]. The performance of seizure detection algorithms gets degraded due to the presence of these artifacts in the EEG signals. So, the removal of these artifacts from EEG signals is essential for the proper diagnosis and treatment by the physicians. The common clinical practice is to remove the entire segment of data affected by the artifacts, that may also lead to loss of the relevant information. Moreover, in real-time signal processing applications, as the manual artifact extraction is not possible, discarding segment of data is not conceivable. In such conditions, it is a challenging task to automate the artifact separation procedure [1] [2].

Low-pass filters are used more generously to eliminate EMG artifacts from the EEG signals. However, the frequency spectrum of muscle antiquities signal overlaps with the intersecting EEG signals, low-pass filters suppress the muscle artifacts but at the same time they also abolish the required valuable information.In some of the previous works artifacts are removed from single channel EEG signal using SSAICA [3] which gives the best results for single channel EEG signals. "Some of the latest methods for the elimination of EMG artifacts from EEG signals are Optimal wavelet transform and Surrogate-Based Artifact Removal" [4] [5]. "The canonical correlation analysis (CCA) method was proposed to separate EMG artifacts from the multi-channel EEG signals" [6]. "Unlike the ICA and other wavelet transform techniques, this method estimates the sources based totally on the second order statistics (SOS), which exhibits low computational complexity". Some of the works discussed in [5] [7] are used to remove different artifacts from multi-channel EEG signals. In [9], ICA has been used to eliminate antiquities from the single channel EEG signal. In any case, this methodology isn't important for EEG signal assessment because of the confinements, for example, sources to be separated should be fixed and the frequencies of the source signals should be disjoint. To place in power ICA on single-channel signals, right off the bat mapping of single-channel signal into a multichannel signal is required. In [8] authors utilized "inter channel dependence information to eliminate muscle artifacts in few- 
channel situation by combining multivariate empirical mode decomposition and canonical correlation analysis (MEMDCCA)" which removes EMG artifacts at some limited channels and moreover increases complexity.

Most of the ICA based EEG artifact separation techniques reported above either rely on only one stage or employs a more complex separation procedure. Moreover, overall performance of ICA relies upon on the length of the segment due to the fact that when the larger segment is processed, the greater is the likelihood that the high-quality number of sources overcomes the number of channels (over complete ICA). In this case, ICA will now not be capable to effectively separate the artifact from the neural aspect due to the truth that some beneficial residual EEG facts is lost.

In this paper, we have introduced an effective strategy known as Multi-channel Singular Spectrum Analysis (MSSA) to take out EMG artifacts from the multi-channel EEG signals. In MSSA, right off the bat the symmetrical eigenvectors of multi-channel information are assessed by the Singular Value Decomposition (SVD). To take out the intriguing component, we have assessed each eigenvector by setting a legitimate pinnacle recurrence limit, the information matrix (G) was planned onto the region stretched out by the eigenvectors whose recurrence is significantly less than the pre-indicated edge recurrence. Finally, the remedied EEG signal is extricated with the guide of performing reverse implanting and the diagonal averaging on the information matrix $(\hat{\mathbf{G}})$. The proposed procedure is approved by the utilization of randomly generated sinusoidal signals debased with the random noise and continuous ictal EEG signals tainted by EMG artifacts. Recreation results show that the proposed approach dispenses EMG antiquities successfully as opposed to the current methods.

\section{Methodology}

\section{A. Multi-channel SSA}

The SSA technique involves two complementary stages: (i) Decomposition and (ii) Reconstruction. The contaminated EEG signal $\left(Y_{n}\right)$ is decomposed at the first stage and the noise compressed EEG $\left(X_{n}\right)$ is reconstructed at the second stage.

1) Decomposition: The decomposition stage of SSA its further divided into two sub-steps embedding and Singular Value Decomposition (SVD). The basic difference between MSSA and SSA is that in MSSA embedding step is not required, since embedding step is used to map single channel signal into multi-channel signal or data. Consider a multichannel data matrix $(\mathbf{G})$ of size $(K \times M)$ as shown below, where $(K=N-L+1), \mathrm{N}$ represents number of Samples and $\mathrm{L}$ represents the Length of the window.

$$
\mathbf{G}=\left[\begin{array}{ccccc}
G(1) & G(2) & \ldots & \ldots & G(K) \\
G(2) & G(3) & \ldots & \ldots & G(K+1) \\
\vdots & \vdots & \ldots & \ldots & : \\
G(M) & G(M+1) & \ldots & \ldots & G(N)
\end{array}\right]
$$

In the following stage SVD is performed on the information matrix G. The SVD of information matrix is given as $\mathbf{G}=\mathbf{V D U}^{T}$, where $\mathbf{V}$ and $\mathbf{U}$ are unitary networks and $\mathbf{D}$ is a diagonal matrix. For the most part, SVD is performed to locate the symmetrical eigen vectors. The covariance matrix $\mathbf{C}$ for information matrix $\mathbf{G}$ is given by $\mathbf{C}=\mathbf{G G}^{T}$, which speak to the relating eigenvalues and eigenvectors as $\lambda_{1}, \lambda_{2}, \ldots, \lambda_{M}$ and $\mathbf{V}_{1}, \mathbf{V}_{2}, \ldots, \mathbf{V}_{M}$ individually. The $i_{t h}$ component of $\mathbf{U}$ can be spoken to as in (1),

$$
\mathbf{u}_{i}=\frac{\mathbf{G}^{T} \mathbf{v}_{i}}{\sqrt{\lambda_{i}}}
$$

where $i=1,2, . ., M$. Then, the trajectory matrix can be expressed as,

$$
\mathbf{G}=\sum_{i=1}^{M} \mathbf{G}_{i}=\sum_{i=1}^{M} \sqrt{\lambda_{i}} \mathbf{v}_{i} \mathbf{u}_{i}^{T}
$$

where trajectory matrix (TM) $\mathbf{G}_{i}$ can be defined as

$$
\mathbf{G}=\mathbf{v}_{i} \mathbf{v}_{i}^{T} \mathbf{G}
$$

The term $\mathbf{v}_{i} \mathbf{v}_{i}^{T}$ in (3) forms a subspace, which is formed by the eigenvector $\mathbf{v}_{i}$.

2) Reconstruction: This reproduction phase of SSA comprises two steps namely, grouping and diagonal averaging.

Grouping: In this progression from the pattern of eigenvalues, the ideal subspace having a place with the signs of intrigue and the undesired subspace having a place with the ancient rarities are isolated. This frequently requires a few ideas regarding the ideal segments. The grouping step relates to parting the basic networks into a few groups and adding the lattices inside each grouping. Let the lists are $I=(1,2,3, \ldots, M)$ which are part into $L$ number of groups; and is performed dependent on the magnitude of the eigenvalue and chosen physically. The trajectory matrix $(\mathrm{G})$ is represented as

$$
G=\sum_{j=1}^{L} G_{I_{j}}
$$

where $G_{I_{j}}$ is the TM and $I_{j}$ represents set of indices for $j^{\text {th }}$ group with $j=1,2, \ldots, L$. Of the chance that $L=M$, at that point the grouping is referred to as elementary grouping.

Diagonal averaging: The reason for inclining averaging is to recreate a specific component or sign from the measured signal, radically change the corresponding TM to a Hankel matrix which can, as a result, be converted to an original signal, which can be subsequently converted to the original. For example, to expel the extraordinary imperativeness segment from the conscious sign, right off the bat, the related TM is formed by introducing the information matrix $(\mathrm{G})$ onto the subspace stretch with eigenvectors comparing to the huge eigenvalues. To expand the supported subspace, the choice of eigenvectors reliant on the degrees of the eigenvalues are not reasonable for the capacity, for instance, signals polluted by the unpredictable 

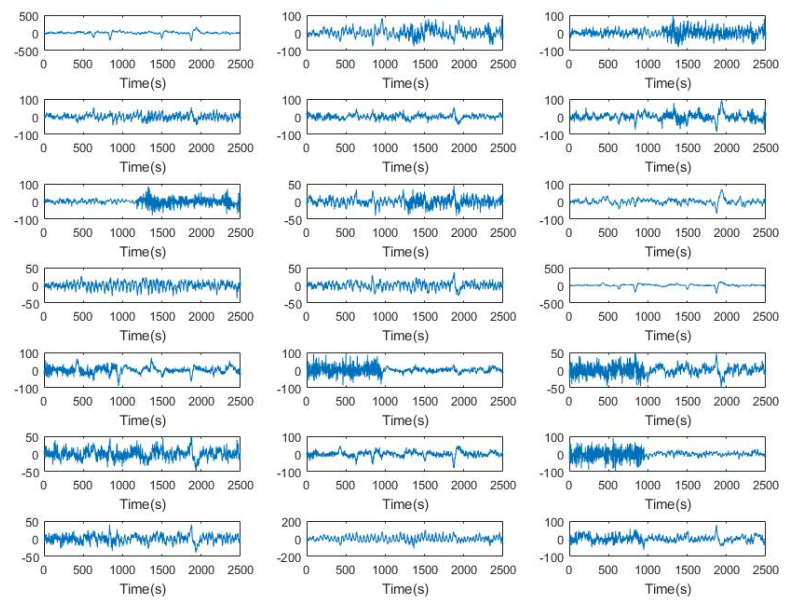

Fig. 1. 21-channels contaminated ictal EEG data.

upheaval. In this paper, to perceive the supported subspace the eigenvectors required to broaden the subspace are picked depending on their peak frequencies then again of the extents of the eigenvalues.

\section{B. Calculation of Peak Frequency}

Peak frequency is simply the frequency of maximum power signal. For pure EEG signals embedded with some EMG artifacts this technique often provide the best pitch estimate. The peak frequency is observed in low to medium muscle contractions is present in the $\beta$-band $(13-30 \mathrm{~Hz})$. The peak frequency during the high muscle contractions is noticed in the $\alpha$-band. This is referred to as the piper rhythm, wherein coherence is observed at $(30-60 \mathrm{~Hz})$ during muscle release. It can be calculated by determining the frequency response of a digital filter designed to filter signals sampled at frequency $f_{s}$. Frequency response of digital filter is given as,

$H(z)=\frac{B(z)}{A(z)}=\frac{b(1)+b(2) z^{-1}+\ldots b(n+1) z^{-n}}{a(1)+a(2) z^{-1}+\ldots a(n+1) z^{-m}} \mid z=e^{-j \omega}$

Since we have determined the peak frequency in MATLAB, we utilized the function freqz to assess the frequency response. At the point when a vector of frequencies is provided as an information contention, at that point freqz assesses the polynomials at every frequency point utilizing Horner's strategy for settled polynomial assessment, separating the numerator response by the denominator response as in (5). At

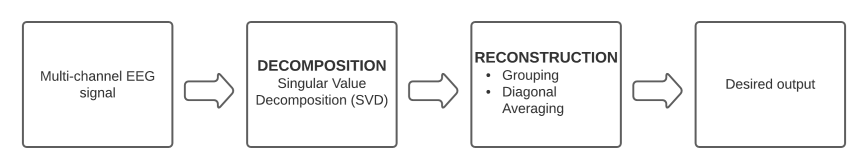

Fig. 2. Proposed method
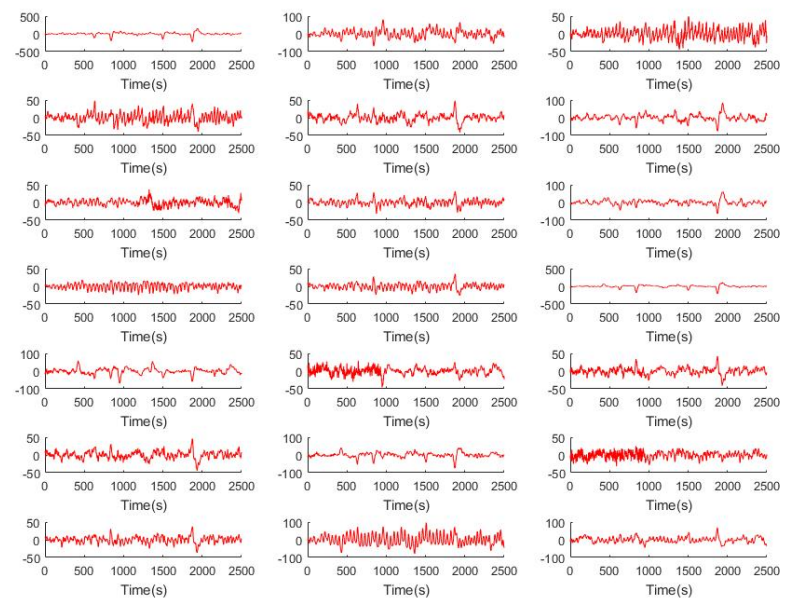

Fig. 3. 21-channels corrected ictal EEG data by MSSA technique.

long last, the frequencies over the assigned limit are eliminated to get an artifact-free EEG signal.

\section{Proposed MSSA Technique}

In this paper, we have presented a replacement grouping technique that is implemented using the MSSA algorithm as shown in Fig 2. The concept of this proposed technique is thee eigenvectors which are wont to extend the specified subspace, the eigenvalues are selected supported their peak frequencies rather than the magnitude. This system is incredibly efficient in recognizing EEG signals which are contaminated with EMG artifacts. Consider $G(n)$ and $A(n)$ as desired and contaminated statistically unconventional source signals which are originated at $g_{0}$ and $g_{1}$, respectively. Then, the measured mono-channel EEG signal can be represented by

$$
X(n)=G(n)+\rho A(n)
$$

where $\rho$ is a propagation constant, which decides the role of a particular contaminated signal in $X(n)$.

To extricate the spotless EEG signal from the ruined EEG signal we have assessed the peak frequencies of each eigenvector. Subsequent to evaluating peak frequencies the information matrix $\mathbf{G}$ was anticipated onto the subspace, the eigenvectors having peak frequencies not exactly the assigned limit, have a place with the TM of the EEG signal. Finally, diagonal averaging has been performed on the TM to extricates the multi-channel antiquity free EEG signal.

\section{RESUltS ON SYNTHETIC DATA}

To present the validity of the proposed MSSA technique, we have considered the signal mixed with some random noise represented in (6). SNR value of the signal is given by

$$
S N R=\frac{R M S(G)}{R M S(\rho A)}
$$



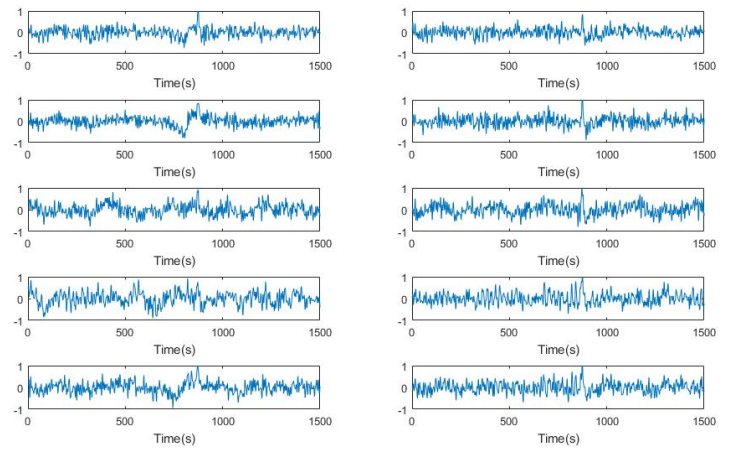

Fig. 4. 10-Channels Contaminated Synthetic EEG data.

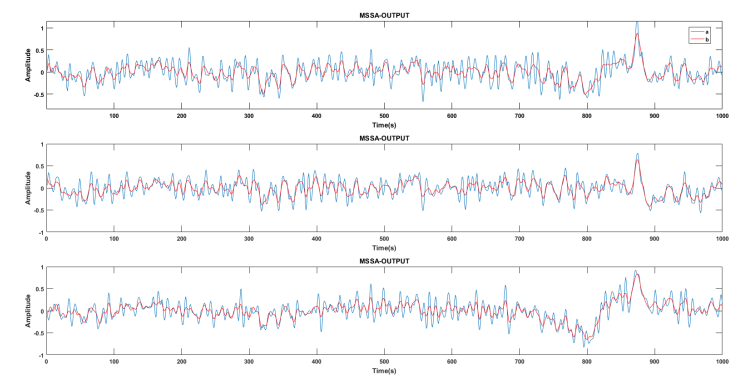

Fig. 5. 3-Channels MSSA output.

Here, $\rho$ represents the SNR value of the measured signal $X(n)$.

To compare the performance of the proposed MSSA technique with the existing CCA technique, we have considered the relative root-mean-square error (RRMSE) as a error parameter between orginal signal and artifact free signal. The RRMSE of the required source signal $G(n)$ is given by

$$
R R M S E=\frac{R M S(G-\hat{G})}{R M S(S)} \times 100 \%
$$

Where $\mathbf{G}$ is the original data matrix and $\hat{\mathbf{G}}$ is data matrix after diagonal averaging.

\section{A. Extraction of EEG Signal Using MSSA Technique}

At first, the proposed technique is applied on 21-channel ictal EEG data to observe the performance of proposed technique in removing EMG artifacts Fig. 1. The outputs of the ictal EEG data are shown in Fig. 3.

Later, the proposed method is applied on the 10-channel synthetic EEG signal which was tainted by EMG artifact shown in Fig. 4. The multi-channel EEG During Mental Arithmetic Tasks recording of Subject001edfm was taken from physionet database [10]. We considered 10-channels from the 19-channels data with a sampling frequency of $500 \mathrm{~Hz}$. The

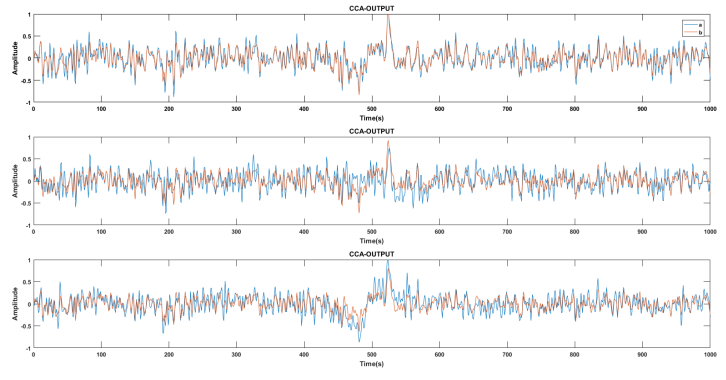

Fig. 6. 3-Channels CCA output.

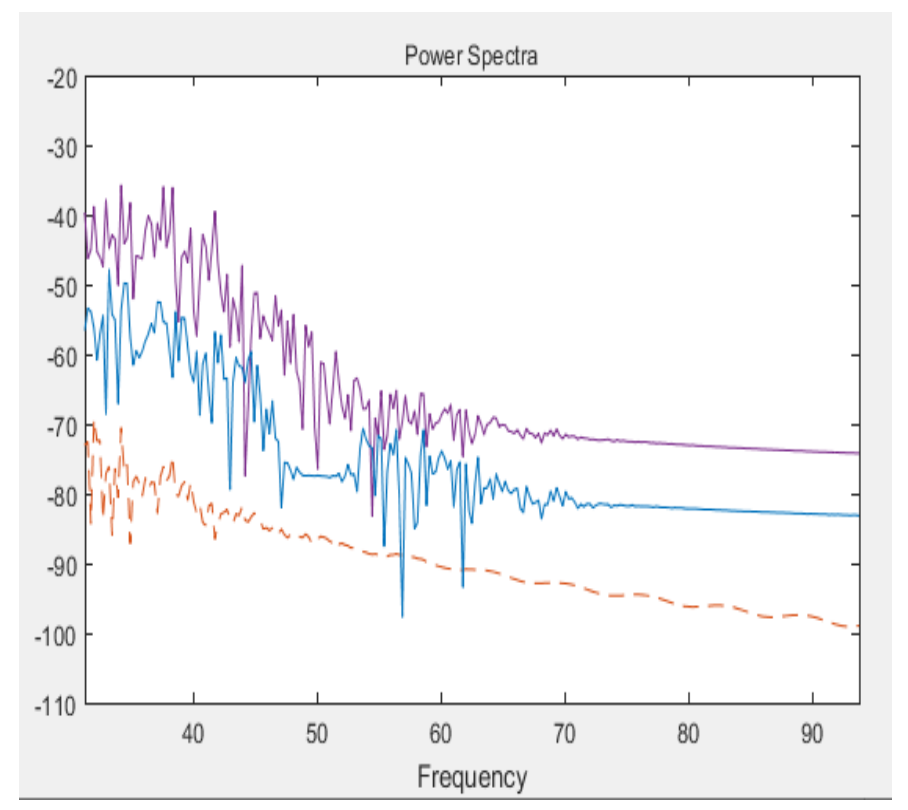

Fig. 7. PSD output of original EEG (blue), Corrupted EEG (purple) and Purified EEG (dotted)

proposed MSSA technique is then applied on the 3-channel synthetic EEG data corrupted with EMG artifacts. During simulation the sampling frequency was reduced to $250 \mathrm{~Hz}$, window size was set to 40 during entire simulation and the maximum peak frequency threshold was set to $21 \mathrm{~Hz}$ since all the EMG artifacts fall under $(13-30 \mathrm{~Hz})$. The artifact corrected EEG data after using MSSA technique is shown in Fig. 5. PSD graph is plotted between orginal EEG, noisy EEG and purified EEG as shown in Fig. 7. Then by varying propagation constant $\rho$ we have plotted the RRMSE curves between the original EEG data and the contaminated EEG data. Then, the data is used to simulate using CCA technique to remove EMG artifacts as shown in Fig. 6. Finally, both the RRMSE and RMSE curves were compared to show that the proposed MSSA technique outperforms the CCA technique in removing EMG artifacts from EEG signals shown in Fig. 8 and Fig. 9.

\section{CONCLUSION}

In this paper, we have introduced an MSSA-based strategy for the expulsion of EMG antiques from the EEG signals. We 
TABLE I

COMPARISON OF RRMSE AND RMSE VALUES BETWEEN PROPOSED MSSA AND CCA TECHNIQUES

\begin{tabular}{|l|l|l|l|l|}
\hline \multirow{2}{*}{$\begin{array}{l}\text { SNR } \\
(\mathbf{d B})\end{array}$} & \multicolumn{2}{|c|}{ RRMSE(\%) } & \multicolumn{2}{c|}{ RMSE(\%) } \\
\cline { 2 - 5 } & $\boldsymbol{M S S \boldsymbol { A }}$ & $\boldsymbol{C C A}$ & MSSA & $\boldsymbol{C A}$ \\
\hline-9.87 & 88.69 & 157.90 & 0.1616 & 0.2113 \\
\hline-8.84 & 83.10 & 139.31 & 0.1457 & 0.1919 \\
\hline-7.70 & 73.73 & 121.66 & 0.1254 & 0.1757 \\
\hline-6.42 & 63.26 & 104.24 & 0.1236 & 0.1840 \\
\hline-5.00 & 55.06 & 93.89 & 0.1015 & 0.1673 \\
\hline-3.45 & 46.78 & 74.03 & 0.0809 & 0.1360 \\
\hline-1.86 & 35.56 & 58.31 & 0.0647 & 0.1072 \\
\hline-0.53 & 26.85 & 35.46 & 0.0488 & 0.0617 \\
\hline
\end{tabular}

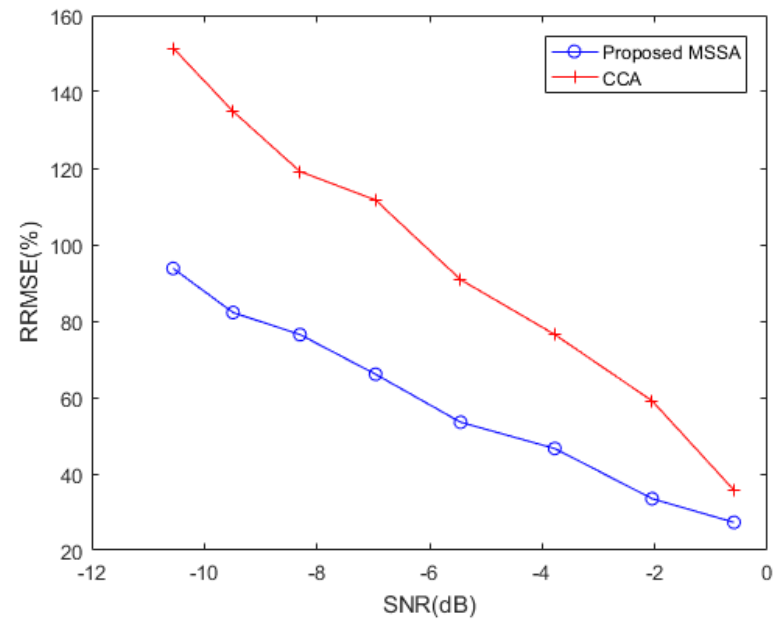

Fig. 8. Comparison of RRMSE curves between CCA technique and the proposed MSSA techniques.

have thought about the aftereffects of the proposed method with the current CCA procedure. By assessing the exhibitions of two methodologies as demonstrated in Table I, it could be confirmed that the MSSA-based methodology got more noteworthy SNR and substantially less RRMSE and RMSE values than the CCA-based technique. Contrasted with the CCA-based strategy, the MSSA-based procedure is less difficult to expel the EMG antiques from the EEG signals, hence this method is more noteworthy proper to wipe out the EMG antiques from the EEG signals. Later on, the MSSA-based methodology can be utilized for the handling of ongoing EEG signals so as to comparably certify the adequacy of the EMG antiquity end of the proposed strategy.

\section{ACKNOWLEDGEMENT}

This work was carried out as a part of summer research fellowship at Indian Institute of Technology, Guwahati, we as authors would like to thank IIT Guwahati for his guidance throughout the work.

\section{REFERENCES}

[1] A. K. Maddirala and R. A. Shaik, "Removal of EMG artifacts from single channel EEG signal using singular spectrum analysis," 2015 IEEE International Circuits and Systems Symposium (ICSyS), Langkawi, 2015, pp. 111-115, doi: 10.1109/CircuitsAndSystems.2015.7394075.

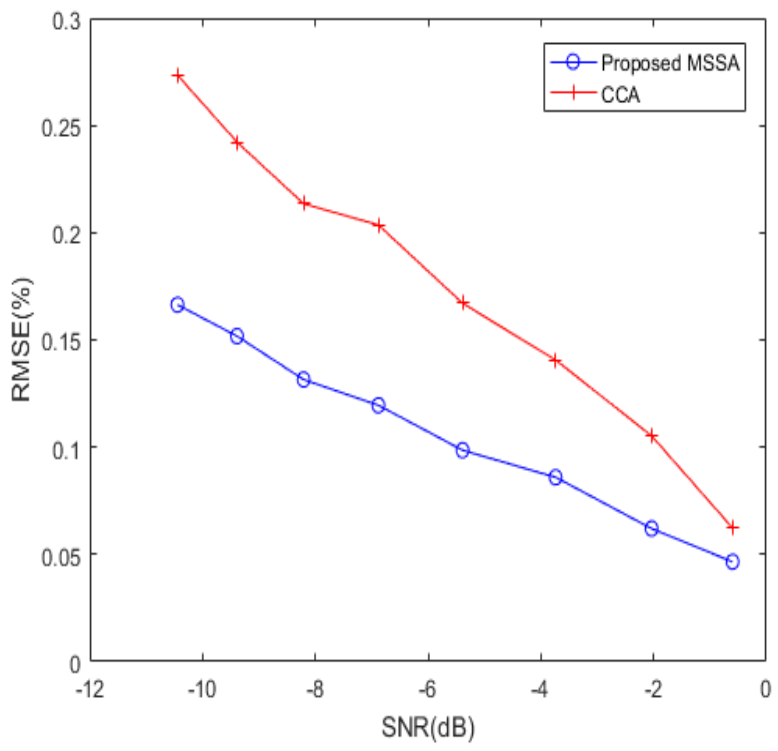

Fig. 9. Comparison of RMSE curves between Proposed MSSA technique and CCA technique

[2] N. Mammone, F. La Foresta and F. C. Morabito, "Automatic Artifact Rejection From Multichannel Scalp EEG by Wavelet ICA," in IEEE Sensors Journal, vol. 12, no. 3, pp. 533-542, March 2012, doi: 10.1109/JSEN.2011.2115236

[3] A. K. Maddirala and R. A. Shaik, "Separation of Sources From SingleChannel EEG Signals Using Independent Component Analysis," in IEEE Transactions on Instrumentation and Measurement, vol. 67, no. 2, pp. 382-393, Feb. 2018, doi: 10.1109/TIM.2017.2775358.

[4] Z. A. A. Alyasseri, A. T. Khader, M. A. Al-Betar, A. K. Abasi and S. N. Makhadmeh, "EEG Signals Denoising Using Optimal Wavelet Transform Hybridized With Efficient Metaheuristic Methods," in IEEE Access, vol. 8, pp. 10584-10605, 2020, doi: 10.1109/ACCESS.2019.2962658.

[5] M. Chavez, F. Grosselin, A. Bussalb, F. De Vico Fallani and X. Navarro-Sune, "Surrogate-Based Artifact Removal From Single-Channel EEG," in IEEE Transactions on Neural Systems and Rehabilitation Engineering, vol. 26, no. 3, pp. 540-550, March 2018, doi: 10.1109/TNSRE.2018.2794184.

[6] W. D. Clercq, A. Vergult, B. Vanrumste, W. Van Paesschen, and S. Van Huffel, "Canonical correlation analysis applied to remove muscle artifacts from the electroencephalogram," IEEE Transactions on Biomedical Engineering, vol. 53, no. 12, pp. 2583-2587, Nov 2006.

[7] K. P. Paradeshi, R. Scholar and U. D. Kolekar, "Removal of ocular artifacts from multichannel EEG signal using wavelet enhanced ICA," 2017 International Conference on Energy, Communication, Data Analytics and Soft Computing (ICECDS), Chennai, 2017, pp. 383-387, doi: 10.1109/ICECDS.2017.8390150.

[8] X. Chen, X. Xu, A. Liu, M. J. McKeown and Z. J. Wang, "The Use of Multivariate EMD and CCA for Denoising Muscle Artifacts From Few-Channel EEG Recordings," in IEEE Transactions on Instrumentation and Measurement, vol. 67, no. 2, pp. 359-370, Feb. 2018, doi: 10.1109/TIM.2017.2759398.

[9] M. Davies and C. James, "Source separation using single channel ICA," Signal Processing, vol. 87 , no. 8, pp. $1819-1832$

[10] https://archive.physionet.org/cgi-bin/atm/ATM 